Stefano Barbieri

Marco Serena

\title{
Winner's Effort Maximization in Large Contests
}

Max Planck Institute for Tax Law and Public Finance Working Paper 2020 - 13

September 2020

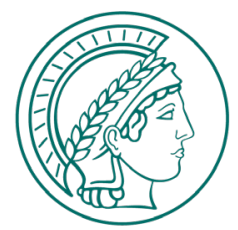

Max Planck Institute for Tax Law and Public Finance

Department of Business and Tax Law

Department of Public Economics

http:/ / www.tax.mpg.de 
Working papers of the Max Planck Institute for Tax Law and Public Finance Research Paper Series serve to disseminate the research results of work in progress prior to publication to encourage the exchange of ideas and academic debate. Inclusion of a paper in the Research Paper Series does not constitute publication and should not limit publication in any other venue. The preprints published by the Max Planck Institute for Tax Law and Public Finance represent the views of the respective author(s) and not of the Institute as a whole. Copyright remains with the author(s).

Max Planck Institute for Tax Law and Public Finance Marstallplatz 1

D-80539 Munich

Tel: $\quad+498924246-0$

Fax: $\quad+498924246-501$

E-mail:ssrn@tax.mpg.de

http://www.tax.mpg.de 


\title{
Winner's Effort Maximization in Large Contests
}

\author{
Stefano Barbieri*, Marco Serena ${ }^{\dagger}$
}

April 29, 2020

\begin{abstract}
We investigate the temporal structure that maximizes the winner's effort in large homogeneous contests, thus extending Hinnosaar (2019)'s analysis of total effort. We find that the winner's effort ranges from a lower bound of 0 to an upper bound of one third of the value of the prize, depending on the temporal structure; the upper (lower) bound is reached with an infinite number of players playing sequentially (simultaneously) in the first periods (period). This is in line with Hinnosaar's results for total effort. Nevertheless, when compare the speed of convergence of different temporal structures to the upper bound for the winner's effort, we prove the suboptimality of the fully sequential temporal structure, which is dominated by an initial number of fully sequential moves and a greater number of fully simultaneous moves in the very last period. This is in contrast with Hinnosaar's results for total effort.
\end{abstract}

\section{Introduction}

In many applications of sequential contests, it is natural to ask whether the efforts of earlier players should be disclosed to later players or not. For instance, in R\&D contests, firms' investments may or may not be disclosed to the other competing firms by a regulator. If a regulator chooses to disclose firms' investments, later movers can tailor their investments to the early movers' choices. This dynamic effect is absent should the regulator choose not to disclose investments instead.

\footnotetext{
*Tulane University. Email: sbarbier@tulane.edu

${ }^{\dagger}$ Max Planck Institute for Tax Law and Public Finance. Email: marco.serena85@gmail.com
} 
The elegant characterization of Hinnosaar (2019) addresses the above question in a sequential Tullock contest with $n$ homogeneous players whose efforts may or may not be disclosed to later contestants. Note that the choice of disclosure policy when players move sequentially can be equivalently stated as a choice of temporal structure under complete information; in fact, full disclosure (concealment) in the former setup coincides with a fully sequential (simultaneous) temporal structure in the latter setup. Hence, Hinnosaar (2019)'s result can be rephrased, under complete information, as the optimality of a fully sequential temporal structure among all possible temporal structures - simultaneous or (partially) sequential.

We borrow Hinnosaar (2019)'s Tullock-contest setup with $n$ homogeneous players, and we focus on investigating how the temporal structure affects the expected winner's effort (henceforth, $W E$ ), rather than the expected total effort (henceforth, $T E$ ), which is the focus of Hinnosaar (2019)'s contribution. $W E$ is an objective of particular interest in contests, and its analysis complements the one of $T E$. In an R\&D contest, for instance, the effort exerted in developing the winning project is often the only ex-post valuable effort to the contest designer, as the winning project, rather than on the losing project(s), typically obtains founding, market share, visibility and is eventually implemented. Hence, weighting each contestant's effort by its probability of winning the contest is a natural objective when the designer cares about the quality of the winning project only (i.e., $W E$-maximization), rather than about stimulating participants' efforts in general (i.e., $T E$-maximization).

Nevertheless, moving from Hinnosaar's analysis of $T E$ to our analysis of $W E$ in Tullock contests entails a two-fold loss of tractability. In fact, first, while in $T E$ efforts are all equally weighted, in $W E$ each effort is weighted by the individual's own probability of victory, which in turn depends on all other efforts. And second, Hinnosaar (2019)'s innovative inverted best-response approach $^{1}$ allows a characterization of $T E$ by leveraging the fact that in Tullock contests a player's payoff depends only on her own effort and $T E$ itself; this makes the analysis of $W E$ more challenging than that of $T E$, especially as we build on Hinnosaar (2019)'s characterization results. We show in Subsection 3.1 that, among the five key simplifying properties that Hinnosaar shows to hold for $T E$, none carries over to $W E$.

We sidestep the challenge posed by the above-mentioned loss of tractability

\footnotetext{
${ }^{1}$ Hinnosaar (2019) builds on this approach as the standard backward induction fails in this class of games. For details, see Appendix C of Hinnosaar (2019).
} 
in the analysis of $W E$ by analyzing large contests (where the number of players $n \rightarrow \infty)$, which restore a great deal of tractability. Hence, this brings our contribution closer to the literature on large contests; see, for instance, the seminal contribution by Olszewski and Siegel (2016) and also Bodoh-Creed and Hickman (2018) and Olszewski and Siegel (2020a, 2020b, 2020c). Our results in Section 4 analyze $W E$ by building on Hinnosaar (2019)'s characterization results for large contests.

Our $W E$-maximizing results for large contests in part confirm Hinnosaar's $T E$-maximizing results, and in part reveal some differences. While Hinnosaar (2019) shows that in large contests $T E$ converges to 1 regardless of the temporal structure, we find that $W E$ converges to a value in the interval $[0,1 / 3]$, depending on the temporal structure. We fully characterize the temporal structures whose $W E$ converges to one of the two extreme values 0 and $1 / 3$. The lower bound 0 is reached by temporal structures with an infinite number of players playing simultaneously in the first period. The upper bound $1 / 3$ is reached by temporal structures with an infinite number of players playing sequentially in the initial periods. These two results resemble Hinnosaar's results for $T E$ for finite $n$ : $T E$ is maximum (minimum) under a fully sequential (simultaneous) temporal structure. Nevertheless, the upper bound $1 / 3$ is reached by a large number of temporal structures of large contests. Hence, we compare the speed of convergence to $1 / 3$ of different $W E$-optimal temporal structure, and we characterize a number of strict rankings of temporal structures. We find that for any temporal structure with at least one period with infinite simultaneous plays there is a temporal structure with finite plays in every period (and hence infinite periods) yielding greater $W E$. Moreover, we find that a fully sequential temporal structure is dominated by a sequential temporal structure with some simultaneous moves in the last period, in contrast with Hinnosaar's TEmaximizing results. More generally, we find that in the family of contests where in the very last period a number of players play simultaneously and in the initial periods players play sequentially, it is $W E$-maximizing to have more players play simultaneously than sequentially, once again in contrast with Hinnosaar's $T E$-maximizing results. This shows the suboptimality of a fully sequential temporal structure when maximizing $W E$, which is in contrast with Hinnosaar's results for total effort.

Comparison with Hinnosaar (2020) and maximization of the highest effort. The independent work Hinnosaar (2020) extends Hinnosaar (2019)'s 
analysis to a richer set of possible objectives for the contest designer, other than $T E$. He does not consider $W E$ though. Among the objectives he considers, the closest in nature to $W E$ is the highest effort $(H E)$. In this section, we describe the different interpretation between $W E$ and $H E$, and then the different results obtained by Hinnosaar (2020) for $H E$ and by us for $W E$.

As for the interpretation, maximization of $H E$ in a Tullock contest could be interpreted as maximizing the quality of the best entry in say an R\&D contest, but, because of the noise inherent in the Tullock contest success function, the best entry could end up losing in a Tullock contest and, if this is the case, its quality would be of no value to a designer interested in maximizing the quality of the winning entry. Maximization of $W E$ takes such a designer's viewpoint into account by weighting each effort by its probability of winning the contest.

As for the results, since the first mover's effort is always the greatest one (i.e., it equals $H E$ ) regardless of the rest of the temporal structure, $H E$-maximization focuses on maximizing the first-mover's effort. This implies, as shown by Hinnosaar (2020), that for a sufficiently low number of players $n$ the $H E$-maximizing contest is one with a single mover and followers arranged in pairs, while for large contests any temporal structure with a first-mover ensures maximal $H E$. We instead find that for both sufficiently low $n$ and large contests the optimal contest takes the form of an initial series of players playing sequentially, and some players playing simultaneously in the last period(s). In fact, for instance, we find that within the family of large contests with a number of initial sequential movers and a number of last simultaneous moves the former number is lower than the latter in the optimum - that is, more players play simultaneously than sequentially (see Proposition 11).

When maximizing $W E$, there are two extra forces at play than when maximizing $H E$. First, in $W E$, the first-mover's effort is weighted by the probability that she wins, rather than by 1 , and such a probability depends on everyone else's effort too. Second, everyone else's (strictly positive) effort has a chance of winning, and thus plays a role too in $W E$. As clear from the explanation in Subsection 3.2, the first of such extra forces is behind the difference between Hinnosaar's $H E$-maximizing and our $W E$-maximizing results. Note that, even if tempting, $W E$ could not simply be seen as a maximization of the probability that the highest effort wins, because such a probability is maximized when all other players exert no effort, hence discouraging competition and reducing the highest effort itself. 
The structure of the paper is as follows. In Section 2 we present some preliminary results and intuitions about $W E$, useful for the subsequent analysis. In Section 3 we build intuition analyzing sufficiently low number of players $n$; Subsection 3.1 shows that $n=4$ suffices to see that Hinnosaar's results and key properties of $T E$ do not carry over to $W E$, Subsection 3.2 builds on the $n=4$ case to deliver the main intuitions, and Subsection 3.3 extends the results analytically to $n=5$, and numerically to $n \geq 6$, but low. The main contribution of the paper is Section 4, where we provide a number of results in large contests $(n \rightarrow \infty)$ on upper and lower bounds for $W E$, on which temporal structures achieve these bounds, and about the speeds of convergence to the upper bound of different temporal structures. Our results for large contests confirm the main results of Section 3 derived for low $n$.

\section{Preliminary results for $W E$}

We adopt Hinnosaar (2019)'s model. Each of $n$ contestants chooses effort $x_{i} \geq 0$ with $i \in\{1, . ., n\}$. A prize of value 1 is given to a contestant according to the Tullock success function; player $i$ 's probability of obtaining the prize equals $x_{i} / X$, where $X$ is the sum of all contestants' efforts. Marginal effort costs are unitary. A designer chooses the temporal structure (or, equivalently, the disclosure policy), denoted by $\mathbf{n}=\left(n_{1}, n_{2}, ..\right) . n_{1}$ contestants simultaneously exert effort in the first period. Their efforts are then publicly disclosed, and $n_{2}$ contestants simultaneously exert effort in the second period. And so on.

For consistency with Hinnosaar's notation, henceforth, we denote by $X$ expected total effort (rather than by TE). From Theorem 1 in Hinnosaar (2019), we know that equilibrium individual effort is ${ }^{2}$

$$
x_{i}=\frac{f_{t}(X)-f_{t-1}(X)}{n_{t}}, \text { with } i \in \mathcal{I}_{t}
$$

where $n_{t}$ is the number of players playing simultaneously in period $t$, and from (7) in Hinnosaar that the recursive definition of $f$ follows

$$
f_{t-1}(X)=f_{t}(X)-n_{t} f_{t}^{\prime}(X) * X(1-X) .
$$

From (1) and (2) one can write the equilibrium probability of victory of

\footnotetext{
${ }^{2}$ All quantities are in equilibrium, hence we omit any indicator of equilibrium quantity to lighten notation throughout the paper.
} 
player $i \in \mathcal{I}_{t}$ as

$$
p_{i}=\frac{x_{i}}{X}=\frac{f_{t}^{\prime}(X) * X(1-X)}{X}=f_{t}^{\prime}(X) *(1-X) .
$$

Also, we have

$$
\begin{aligned}
W E & =\frac{x_{1}}{X} x_{1}+\frac{x_{2}}{X} x_{2}+. . \\
& =X\left(\frac{x_{1}^{2}}{X^{2}}+\frac{x_{2}^{2}}{X^{2}}+. .\right) \\
& =X\left(p_{1}^{2}+p_{2}^{2}+. .\right) .
\end{aligned}
$$

We can make a first cut into understanding $W E$ using formula (4); $W E$ is the product of total effort $X$ and an Herfindahl-Hirschman index on probabilities. Hence, these two terms complement each other; both a high $X$ with "disperse" probabilities (in the worse case, $p_{i}=p_{j} \forall i, j$ ) or a low $X$ with concentrated distribution of probabilities (in the best case, $p_{i}=1$ and $p_{-i}=0$ ) are less valuable in terms of $W E$ than a balance between the two complementary terms.

From (3) and (4), we obtain

$$
\begin{aligned}
W E & =(1-X)^{2} X \sum_{i=1}^{n}\left[p_{i}\right]^{2} \\
& =(1-X)^{2} X \sum_{t=1}^{T}\left[n_{t} f_{t}^{\prime}(X)\right]^{2} .
\end{aligned}
$$

Notice that (5) is valid for every temporal structure. Hence, the key to rank $W E$ 's is the characterization of the functions $f_{t}$ for every temporal structure. Finally, $X$ is the highest root in $[0,1]$ of $f_{0}(X)$, to be plugged into the expression for $W E$.

The temporal structure $\mathbf{n}=\left(n_{1}, n_{2}, ..\right)$ affects the entire sequence of functions $f_{t}$, and thus a full-ranking of temporal structures for general $n$-player is hard to achieve. ${ }^{3}$ We start with the analysis of simple cases.

For three players, one can easily show that the analyses of $W E$ and of total effort $X$ yield the same ranking; namely, temporal structures for both $W E$ and $X$ are ranked $(1,1,1)>(1,2)>(2,1)>(3)$. In fact, Hinnosaar's main

\footnotetext{
${ }^{3}$ Hinnosaar obtains a full-ranking of contest temporal structures for $X$ in large contests, when $n \rightarrow \infty$. However, for finite $n$, the full-ranking remains an open question for both $X$ and $W E$.
} 
result, derived under great generality, is that information disclosure (i.e., more sequentiality of moves under complete information) increases total effort $X$.

For four players, achieving a full-ranking for $W E$ is still a tractable task; in the next section, we analytically prove that already in the $n=4$ case Hinnosaar's result that more information increases total effort $X$ does not carry over to $W E$, and we show how.

\section{Finite contests}

\section{$3.1 n=4$ : Hinnosaar's key properties do not extend to $W E$}

There are eight possible temporal structures when the contest has four players; namely, $(1,1,1,1),(2,1,1),(1,2,1),(1,1,2),(2,2),(3,1),(1,3),(4)$. For each

such temporal structure, we derive the sequence of functions $f_{t}$ starting from the initial condition $f_{T}(X)=X$, working backward with (2) all the way down to $f_{0}$, and finally deriving the highest root in $[0,1]$ of $f_{0}(X)$, which is the equilibrium $X$.

In this section, we derive the full ranking of the eight temporal structures, focusing on maximization of $X$ in Lemma 1 and of $W E$ in Lemma 2.

Lemma 1 In a four-player contest, the ranking of total effort $X i s^{4}$

$$
\begin{gathered}
X(4)<X(3,1)=X(1,3)<X(2,2)<X(1,1,2) \\
=X(1,2,1)=X(2,1,1)<X(1,1,1,1) .
\end{gathered}
$$

The proof easily follows from Hinnosaar (2019)'s results. Nevertheless, we store it in the Appendix, together with the proofs of the other results in the paper not proven in the main body.

Some of the key properties of total effort spelled out in Corollary 1 of Hinnosaar can be seen in the ranking of Lemma 1. We report here Hinnosaar's properties for total effort $X$, as we will discuss them also later in the context of $W E$-maximization.

\footnotetext{
${ }^{4}$ For the ranking of temporal structures when maximizing $X$ or $W E$ we adopt the notation $X(\mathbf{n})$ and $W E(\mathbf{n})$ for temporal structure $\mathbf{n}=\left(n_{1}, n_{2}, ..\right)$.
} 
1. "If the number of players increases in any particular period or a period with a positive number of players is added, then the total effort increases".

2. "reallocating disclosures in a way that creates a permutation of $n[\ldots]$ does not affect the total effort".

3. "disclosures strictly increase total effort".

4. "more homogeneous contests give higher total effort".

5. "in large contests rents are fully dissipated".

While properties 1 . and 5 . require a change in the number of players, and hence cannot be seen in the exercise of this section where we fix $n=4$, the other three properties already emerge in the $n=4$ case: in the ranking in (6), the equalities illustrate property 2 ., the inequality $X(1,3)<X(2,2)$ illustrates property 4 ., and the remaining inequalities illustrate property 3 ., which is the most important property yielding Hinnosaar's main result $-X$ is maximized by a fully sequential contest and minimized by a fully simultaneous one.

In what follows we will show that none of the five properties of total effort $X$ carries over to $W E$, and that a fully sequential contest can be improved upon.

Using (5), we obtain the full ranking of $W E$ among temporal structures under $n=4$ :

Lemma 2 In a four-player contest, the ranking of $W E$ is

$$
\begin{gathered}
W E(4)<W E(3,1)<W E(2,2)<W E(2,1,1)<W E(1,3) \\
<W E(1,2,1)<W E(1,1,1,1)<W E(1,1,2) .
\end{gathered}
$$

It is clear from the ranking of Lemma 2 that properties 2.-4., which are valid for $X$, do not carry over to $W E$, as can be seen, for instance, in $W E(2,1,1) \neq$ $W E(1,2,1)$ for property 2 ., in $W E(1,1,1,1)<W E(1,1,2)$ for property 3 ., and in $W E(2,2)<W E(1,3)$ for property 4 .. The failure of property 3 . is crucial, as it stands in contrast with Hinnosaar's results that $X$ is maximized by a fully sequential contest; in Subsection 3.2 we provide the intuition behind it.

Properties 1 . and 5 . require a change in the number of players; it is easy to show that property 1 . fails by $W E(2,1)>W E(3,1)$. The large 
contests of property 5 . are more subtle to extend to $W E$, and hence we devote Section 4; while property 5. say that $\lim _{n \rightarrow \infty} X(\mathbf{n})=1 \forall \mathbf{n}$, we find that $\lim _{n \rightarrow \infty} W E(\mathbf{n}) \in[0,1 / 3]$ and there exist temporal structures yielding 0 and temporal structures yielding $1 / 3$. We further characterize which temporal structure n reaches the global maximum $1 / 3$, and compare the speed of convergence of different temporal structures. In a nutshell, we show that Hinnosaar's optimality of a fully sequential contest when maximizing total effort $X$ does not carry over to maximization of winner's effort $W E$ in large contests.

Some easy and tractable cases in between $n=4$ and $n \rightarrow \infty$ are discussed in Subsection 3.3.

\section{$3.2 n=5$ : the intuition for $T E, H E$, and $W E$}

In order to understand the intuition behind the different results for $T E$ in Hinnosaar (2019), for $H E$ in Hinnosaar (2020), and for $W E$ in the present paper, we consider $n=5$ and thoroughly compare the temporal structures $(1,1,1,1,1)$, $(1,2,2)$, and $(1,1,1,2)$, which happen to be the optimal temporal structures for $T E, H E$, and $W E$, respectively. ${ }^{5}$

\begin{tabular}{|l|l|}
\hline$(1,1,1,1,1)$ & Effort \\
\hline Player 1 & 0.442 \\
\hline Player 2 & 0.258 \\
\hline Player 3 & 0.143 \\
\hline Player 4 & 0.076 \\
\hline Player 5 & 0.040 \\
\hline \hline$X$ & 0.959 \\
\hline$W E$ & 0.303 \\
\hline$H H I$ & 0.316 \\
\hline
\end{tabular}

\begin{tabular}{|l|l|}
\hline$(1,1,1,2)$ & Effort \\
\hline Player 1 & 0.444 \\
\hline Player 2 & 0.258 \\
\hline Player 3 & 0.142 \\
\hline Player 4 & 0.051 \\
\hline Player 5 & 0.051 \\
\hline \hline$X$ & 0.946 \\
\hline$W E$ & 0.306 \\
\hline$H H I$ & 0.323 \\
\hline
\end{tabular}

\begin{tabular}{|l|l|}
\hline$(1,2,2)$ & Effort \\
\hline Player 1 & 0.447 \\
\hline Player 2 & 0.177 \\
\hline Player 3 & 0.177 \\
\hline Player 4 & 0.065 \\
\hline Player 5 & 0.065 \\
\hline \hline$X$ & 0.930 \\
\hline$W E$ & 0.291 \\
\hline$H H I$ & 0.313 \\
\hline
\end{tabular}

First, total effort $X$ decreases as we move from $(1,1,1,1,1)$ to $(1,1,1,2)$ to $(1,2,2)$. In fact, as shown under great generality and explained in detail by Hinnosaar (2019), more disclosures increase total effort.

Second, the highest effort $H E$, which regardless of the temporal structure is always the first player's effort (see Hinnosaar's Proposition 3), increases as we

\footnotetext{
${ }^{5}$ Note that, despite $n=4$ is the simplest case where Hinnosaar's results on $X$ do not extend to $W E$, we analyze $n=5$ here because when $n=4$ the optimal temporal structure for $H E$ is either $(1,1,2)$ or $(1,2,1)$.
} 
move from $(1,1,1,1,1)$ to $(1,1,1,2)$ to $(1,2,2)$, as shown by Hinnosaar $(2020)$. In fact, the aggregate effort of all players but the first is $0.517,0.502$, and 0.483 respectively, which is always greater than the effort of the first player, and hence its decrease incentivizes the effort of the first player, as we know from canonical contest theory (i.e., the effort of the first player and the overall effort exerted by the others are strategic substitutes near the equilibrium).

Third, consider the expected winner's effort $W E$, and recall from formula (4) that it can be seen as two terms complementing each other; total effort $X$, and an Herfindahl-Hirschman index $(H H I)$ on probabilities, which we report in the tables above. The temporal structure $(1,2,2)$ achieves the minimum, not only for $X$ as already discussed, but also for the $H H I$; this is mostly driven by the second player, whose effort is much less "disperse", compared to others, than in the other two temporal structures. When comparing $H H I$ between the first two temporal structures, $(1,1,1,2)$ yields a greater $H H \mathrm{I}$; this is mostly due to the last two players exerting a total effort that is in aggregate smaller, though less disperse. When comparing $W E$ between $(1,1,1,1,1)$ and $(1,1,1,2)$, in the complementary effect of $X$ and $H H I$, the increase in $H H I$ dominates the decrease in $X$, thus making $(1,1,1,2)$ achieve the maximum of $W E$.

\section{$3.3 n \geq 6$}

The discussion in Subsection 3.2 tailored the explanation of the intuition to the five-player setting. Numerical simulations with more players show that the key properties of the intuition persist regardless of the number of players. In particular, while total effort decreases when moving from $(1,1, . ., 1,1)$ to $(1,1, . ., 1,2)$, the HHI increases and more than compensates in $W E$ for the decrease in total effort $X{ }^{6}$ The fact that the key property of the above intuition also holds for $n \geq 6$ intuitively suggests that our main results carry over to more general $n$ (greater than 6 and finite).

In fact, when we consider more than 6 players, our numerical simulations show that $W E$ is greater under $(1,1, . ., 1,2)$ than $(1,1, . ., 1,1)$ for any number of players. We used the code provided by Hinnosaar, which compute equilibrium efforts for an arbitrary temporal structure for any $n \leq 12$. Hence, we are agnostic about $n \geq 13$ and finite. The key suboptimality of the fully sequential

\footnotetext{
${ }^{6}$ All these comparisons are for a given fixed $n$. Hence, for instance, we compare $(1,1,1,1,1)$
} to $(1,1,1,2)$. 
temporal structure $(1,1, . ., 1,1)$ also holds in large contests as we will show in Section 4.

Yet, we next show that the temporal structure $(1,1, . ., 1,2)$, despite improving upon $(1,1, . ., 1,1)$, is not the global maximizer of $W E$ among all possible temporal structures, unless $n=4,5$. In fact, when $n=6$, despite $(1,1,1,1,2)$ outperforming the fully sequential contest $(1,1, . ., 1,1)$, the temporal structure $(1,1,1,3)$ outperforms both, and ends up being the global maximizer of $W E$ when $n=6$. This result suggests that the greater is $n$, the larger the number of players that play simultaneously in the last period in the optimal structure; this is consistent with our findings for large contests, where we will show in Section 4 that the optimal $m$ in the family of $(1, . ., 1, m)$ large contests is large and, perhaps surprisingly, larger than the number of initial players playing sequentially (see Proposition 11).

It is challenging to characterize a general full ranking of all temporal structures for any finite, but large, value of $n$. Similarly to Hinnosaar (2019)'s analysis for $X$, we do not provide such general full ranking. Instead, in Section 4, which is the main contribution of this paper, we show that the general lessons learned so far for low $n$ carry over to large contests; the optimal temporal structure is achieved with an initial series of sequential plays and some simultaneous plays in the very last period.

\section{Large contests}

Large contests (i.e., when $n \rightarrow \infty$ ) are particularly tractable. In fact, in this case, Hinnosaar (2019) obtains a full-ranking of contest temporal structures for $X$. Hinnosaar provides in Section 6 the approximation for efforts in large contests. We use them to compare $W E$ in various temporal structures, following the intuition behind the results for finite $n$. The findings of this section suggest the generality of the results found for finite $n$; the optimal temporal structure is achieved with an initial number of fully sequential moves and some players playing simultaneously in the very last period.

Individual efforts are

$$
x_{i}^{*} \approx \frac{1}{\prod_{s=1}^{t}\left(1+n_{s}\right)}
$$


One key difference between total effort $X$ and winner's effort $W E$ is that, while Hinnosaar shows that total effort $X$ converges to 1 (the prize) regardless of the temporal structure, ${ }^{7}$ we find that $W E$ can converge to different values according to the temporal structure. Note that $W E$ in a large contest cannot converge to 1 by definition; if $W E \rightarrow 1, x_{i} \rightarrow 1$ and $x_{-i} \rightarrow 0$ (everyone exerts almost 0 effort while one player, $i$, exerts effort almost equal to the prize). It is immediate to see that, regardless of the temporal structure, this cannot be sustained in equilibrium; every players' payoff would tend to 0 , and hence a unilateral deviation to, for instance, effort $1 / 2$ would be profitable regardless of the temporal structure. Thus, the value at which $W E$ converges, which we define $W E^{\infty}$, is interesting per se, and we dedicate Subsection 4.1 to its analysis. $^{8}$

Then, in Subsection 4.2, we will focus on the temporal structures achieving the maximum value of $W E^{\infty}$, and compare the speed of convergence so as to characterize a further tighter ranking of temporal structures.

\subsection{Asymptotic value of $W E^{\infty}$ of different temporal struc- tures}

We know from Hinnosaar that in large contests $X \rightarrow 1$ regardless of the temporal structure. In this subsection, we focus on the asymptotic value of $W E^{\infty}$. Using (4) and (7), we obtain that in general, when we define $\hat{n} \equiv \sum_{i=1,2, . .} n_{i}$ and $\hat{n} \rightarrow \infty$, we have that

$$
\begin{aligned}
W E^{\infty}\left(n_{1}, n_{2}, n_{3}, . .\right)= & \frac{n_{1}}{\left(1+n_{1}\right)^{2}}+\frac{n_{2}}{\left(1+n_{1}\right)^{2}\left(1+n_{2}\right)^{2}}+ \\
& \frac{n_{3}}{\left(1+n_{1}\right)^{2}\left(1+n_{2}\right)^{2}\left(1+n_{3}\right)^{2}}+\ldots \\
= & \sum_{j=1,2, . .} \frac{n_{j}}{\prod_{l=1}^{j}\left(1+n_{l}\right)^{2}} .
\end{aligned}
$$

In this subsection, we analyze the asymptotic value of $W E^{\infty}$ from three different angles. First, in Lemma 3, Lemma 4, and Proposition 6 we find that $W E^{\infty}$ has a minimum at 0 reached only by an infinite number of simultaneously

\footnotetext{
${ }^{7}$ The temporal structure affects the speed at which the convergence of total effort $X$ occurs. ${ }^{8}$ Formally,$$
W E^{\infty}\left(n_{1}, . ., n_{k}\right) \equiv \lim _{\sum_{j=1,2, . .} n_{j} \rightarrow \infty} W E\left(n_{1}, . ., n_{k}\right) .
$$ 
playing first-movers (including the fully simultaneous large contest), and a maximum at $1 / 3$ reached by an infinite number of initial sequential plays (including the fully sequential large contest). Second, in Proposition 7 we find that it is never optimal to have any period with an infinite number of players playing simultaneously. Third, in Lemma 8 we find that, within the family of temporal structures with at least one period with an infinite number of players, the worst such structure with any number of initial individual players playing sequentially before the period with an infinite number of players yields at least twice as much $W E$ as the best structure with any number of initial pairs (or more) of players playing sequentially. This result stresses once more the optimality of temporal structures with many initial individual players playing sequentially.

\subsubsection{Upper and lower bounds on $W E^{\infty}$}

In a fully simultaneous large contest, we obtain the following.

Lemma 3 In a fully simultaneous large contest $W E^{\infty}(n)=0$.

The intuition is simple. In a fully simultaneous large contest, every players' effort tends to 0 , and only one player wins, and hence the expected effort of the winner tends to 0 too.

In a fully sequential large contest, instead, we obtain the following.

Lemma 4 In a fully sequential large contest $W E^{\infty}(1, . ., 1)=1 / 3$.

In a fully sequential large contest, the first player exerts effort $1 / 2$ and has a probability of victory of $1 / 2$. This implies that $75 \%$ of the resulting $W E$ is entirely accounted for by the first player alone. ${ }^{9}$ This fact highlights once again the key role of the first player in sequential contests, as explained in Subsection 3.2 for the case of finite $n$.

Next, we show that 0 and $1 / 3$ are the minimum and maximum asymptotic values of $W E^{\infty}$ for any temporal structure.

Proposition 5 For any temporal structure, $W E^{\infty}(\mathbf{n}) \in[0,1 / 3]$.

Finally, the next natural question to ask is whether the two extreme values, 0 and $1 / 3$, for $W E^{\infty}$ are reached by any temporal structure other than the fully simultaneous or fully sequential ones. First, the value 0 can be reached only

\footnotetext{
${ }^{9}$ Out of the $W E$ being in total $1 / 3,1 / 4$ is due to the first player.
} 
by a contest with an infinite number of simultaneously playing first-movers; in fact, as long as $n_{1}$ is finite, $W E^{\infty}>0$. Second, the value $1 / 3$ is reached by all temporal structures starting with an infinite number of initial sequential plays. Hence, we obtain the following proposition.

Proposition 6 We have the following,

$$
\begin{aligned}
& W E^{\infty}(\mathbf{n})=0 \Longleftrightarrow n_{1} \rightarrow \infty \\
& W E^{\infty}(\mathbf{n})=1 / 3 \Longleftrightarrow \mathbf{n}=\left(1, . ., 1, m_{1}, . ., m_{k}\right) \text { with }\left(\hat{n}-\sum_{j=1}^{k} m_{j}\right) \rightarrow+\infty .
\end{aligned}
$$

From Proposition 6, for instance, all the temporal structures discussed in Subsection 3.3 brought to the large contest setup yield $W E^{\infty}=1 / 3$, including $(1, . ., 1,2),(1, . ., 1,3)$, and $(1, . ., 1,2,2)$. This remark will inspire most comparisons of speeds of convergence to $1 / 3$ of temporal structures in Subsection 4.2. We will show that, despite $W E^{\infty}(\mathbf{n})$ reaches the maximum value of $1 / 3$ for a large number of temporal structures $\mathbf{n}$, there is a strict ranking between these temporal structures when we compare the speed of convergence of $W E^{\infty}$ to $1 / 3$.

\subsubsection{Finite players in every period}

In general, large contests can be of one of two families: at least one period with an infinite number of players (and a finite or infinite number of periods), or with an infinite number of periods with finite number of players each. In Proposition 7 we find that the former class dominates the latter in terms of $W E^{\infty}$.

First, consider a large contest with at least one period with an infinite number of players. Consider the earliest period, $k \geq 1$, with an infinite number of players. From (8),

$$
\begin{aligned}
W E^{\infty}\left(n_{1}, n_{2}, . ., n_{k-1}, \infty, \ldots\right)= & \sum_{j=1}^{k-1} \frac{n_{j}}{\prod_{l=1}^{j}\left(1+n_{l}\right)^{2}}+\sum_{j=k}^{\infty} \frac{n_{j}}{\prod_{l=1}^{j}\left(1+n_{l}\right)^{2}} \\
= & \sum_{j=1}^{k-1} \frac{n_{j}}{\prod_{l=1}^{j}\left(1+n_{l}\right)^{2}}+\frac{n_{k}}{\prod_{l=1}^{k}\left(1+n_{l}\right)^{2}} \\
& +\frac{1}{\prod_{l=1}^{k}\left(1+n_{l}\right)^{2}} \sum_{j=k+1}^{\infty} \frac{n_{j}}{\prod_{l=k+1}^{j}\left(1+n_{l}\right)^{2}}
\end{aligned}
$$


where the last addend tends to 0 as

$$
\frac{1}{\prod_{l=1}^{k}\left(1+n_{l}\right)^{2}} \sum_{j=k+1}^{\infty} \frac{n_{j}}{\prod_{l=k+1}^{j}\left(1+n_{l}\right)^{2}} \leq \frac{1}{3 \prod_{l=1}^{k}\left(1+n_{l}\right)^{2}} \rightarrow 0
$$

by Proposition 6, and the second addend also goes to 0 as $n_{k} /\left(1+n_{k}\right)^{2} \rightarrow 0$. In words, periods from $k$ onwards do not affect the value of the limit, and $W E^{\infty}$ boils down to

$$
W E^{\infty}\left(n_{1}, n_{2}, . ., n_{k-1}, \infty, \ldots\right)=\sum_{j=1}^{k-1} \frac{n_{j}}{\prod_{l=1}^{j}\left(1+n_{l}\right)^{2}},
$$

which is a finite number in the interval $[0,1 / 3]$ by Proposition $5 .^{10}$

Notice that formula (9) holds as long as at least one period has an infinite number of players, regardless of whether the number of periods is finite or infinite. Thus, we are left to analyze the case of a large contest with finite number of players in every period in what follows.

Second, consider a large contest with an infinite number of periods with finite number of players each. Clearly, for every fixed vector $\left(n_{1}, n_{2}, . ., n_{k-1}\right)$, this family yields a greater $W E^{\infty}$ than the former as

$$
W E^{\infty}\left(n_{1}, n_{2}, . ., n_{k-1}, \infty, \ldots\right)<W E^{\infty}\left(n_{1}, n_{2}, . ., n_{k-1}, n_{k}, \ldots\right)
$$

for every finite $n_{k}$ and every continuation vector of $n_{i}$ 's. Hence, we prove the following:

Proposition 7 For any large contest $\left(n_{1}, n_{2}, ..\right)$ with at least one $n_{i} \rightarrow \infty$, there is a large contest with an infinite number of periods and every $n_{i}$ finite which yields greater $W E^{\infty}$.

\footnotetext{
${ }^{10}$ One could use formula (9) to provide a full ranking of temporal structures for a given value of $k$, and hence, for instance, compute

$$
\begin{aligned}
& W E^{\infty}(1,1, \infty, . .)=\frac{5}{16}>W E^{\infty}(1,2, \infty, . .)=\frac{11}{36}>W E^{\infty}(1,3, \infty, . .)=\frac{17}{64}> \\
& W E^{\infty}(2,1, \infty, . .)=\frac{5}{36}>W E^{\infty}(2,2, \infty, . .)=\frac{10}{81}>W E^{\infty}(2,3, \infty, . .)=\frac{17}{144} .
\end{aligned}
$$
}




\subsubsection{Fully sequential initial periods}

We develop an exercise to quantify the importance of starting the temporal structure with many players playing sequentially so as to obtain high asymptotic values of $W E^{\infty}$. In particular, we show that the worst structure of the form $(\underbrace{1, . .1}_{m_{1}}, \infty, .$.$) with m_{1} \geq 1$ yields at least twice as much $W E$ as the best structure of the form $(\underbrace{a, . ., a}_{m_{2}}, \infty, .$.$) with m_{2} \geq 1$ and $a \geq 2$. In fact, applying (9), we obtain that, for $m_{1} \geq 1$,

$$
\begin{aligned}
W E^{\infty}(\underbrace{1, \ldots, 1}_{m_{1}}, \infty, . .) & =\sum_{k=1}^{m_{1}} \frac{1}{2^{2 k}} \\
& =\frac{2^{-2 m_{1}}\left(2^{2 m_{1}}-1\right)}{3} \\
& \geq \frac{1}{4}
\end{aligned}
$$

which is minimized by $m_{1}=1$, for which $W E^{\infty}=1 / 4$. Similarly, with $m_{2} \geq 1$ and $a \geq 2$,

$$
\begin{aligned}
W E^{\infty}(\underbrace{a, . ., a}_{m_{2}}, \infty, . .) & =\sum_{k=1}^{m_{2}} \frac{1}{a^{2 k}} \\
& =\frac{a^{2 m_{2}}-1}{\left(a^{2}-1\right) a^{2 m_{2}}}
\end{aligned}
$$

which is decreasing in $a$ and increasing in $m_{2}$. Hence, the maximum in this latter class of contest is reached when $a=2$ and $m_{2} \rightarrow \infty$, for which $W E^{\infty}=1 / 8$. Hence, the worst contest in the first class yields twice as much $W E^{\infty}$ as the best contest in the second class. We store this result in the following lemma.

Lemma $8 W E^{\infty}(\underbrace{1, . ., 1}_{m_{1}}, \infty, ..) \geq 2 W E^{\infty}(\underbrace{a, . ., a}_{m_{2}}, \infty, .$.$) for every m_{1}, m_{2} \geq$ 1 , and $a \geq 2$. 


\subsection{Convergence speed of $W E^{\infty}$ under different temporal structures}

In Subsubsection 4.1.1 we showed that $W E^{\infty}$ reaches its maximum value of $1 / 3$ for any temporal structures starting with an infinite number of initial sequential plays. Hence, for instance, all the temporal structures discussed in Subsection 3.3 yield $W E^{\infty}=1 / 3$ in large contests, including for instance $(1, . ., 1,2)$, $(1, . ., 1,3)$, and $(1, . ., 1,2,2)$. However, the analysis of finite $n$ carried out in subsections 3.1 and 3.3 seem to suggest some difference in $W E$ across temporal structure with a sequence of initial sequential plays. In this subsection, we test the lessons learned from the analysis of finite $n$ in the realm of large contests, by comparing the speed of convergence to $1 / 3$ of the temporal structures yielding $W E^{\infty}=1 / 3$. We obtain a battery of results.

Recall first that, in a large contest, $W E$ of the fully sequential temporal structure dominates that of the fully simultaneous temporal structure. However, the former can be further improved upon:

Lemma 9 In a large contest, $W E^{\infty}(1, . ., 1)<W E^{\infty}(1, . ., 1,2)$.

Proof. See the Appendix.

It is then natural to ask whether, within the family of temporal structures $(1, . ., 1, m), m=2$ maximizes the speed of convergence to $1 / 3$. The answer is negative;

Lemma 10 In a large contest, $W E^{\infty}(1, . ., 2)<W E^{\infty}(1, . ., 1,3)$.

Proof. See the Appendix.

Given the two above lemmas, the natural subsequent question is to analyze the optimal $m$ in the family of $(1, . ., 1, m)$ large contests. In particular, comparing temporal structures $(1, . ., 1, m)$ and $(1, . ., 1, m+1)$ for a fixed number of players which tends to infinity, we find that the optimal $m$ is, perhaps not surprisingly, infinite but, perhaps surprisingly, greater than the number of initial sequential plays: ${ }^{11}$

Proposition 11 In the family of large contests $(1, . ., 1, m), W E^{\infty}$ is maximized by making more players play simultaneously than sequentially.

\footnotetext{
${ }^{11}$ Despite Proposition 11 superseding Lemma 10, we provide a direct and independent proof of the latter in the Appendix.
} 
Proof. See the Appendix.

Comparison with findings under finite $n$ 's. A number of results derived in this subsection for large contests support the main findings of the analysis of finite $n$ in Section 3 carry over to large contests with $n \rightarrow \infty$. First, Lemma 9 supports that a fully sequential contest can be improved upon by two simultaneous last-movers, as shown in Section 3 for finite $n$. Second, Lemma 10 supports the optimality of more than two players playing simultaneously in the last period, as shown in Subsection 3.3 for $n=6$.

However, the results of Proposition 11, despite confirming that having some simultaneous last-period movers outperforms a fully sequential contest, it also seems to overturn one lesson drawn from the analysis of finite $n$ 's; namely, while in Section 3 we found that the $W E$-maximizing $m$ in the family of $(1, . ., 1, m)$ contests with $n \in[4,12]$ is smaller than the number of initial sequential plays, in Proposition 11 we found that in large contests, instead, the optimal $m$ is greater than the number of initial sequential plays. A unifying conjecture one may pose to explain both phenomena is that, defining $m^{*}(n)$ to be the $W E^{\infty}$-maximizer within $(1, . ., 1, m)$ contests, the ratio $n / m^{*}(n)$ increases in $n$. 


\section{References}

[1] Bodoh-Creed, A. L., \& Hickman, B. R. (2018). College assignment as a large contest. Journal of Economic Theory, 175, 88-126.

[2] Hinnosaar, T. (2019). Optimal sequential contests. Available at SSRN 3119014.

[3] Hinnosaar, T. (2020). Contest Architecture with Public Disclosures. Available at SSRN 3394708.

[4] Olszewski, W., \& Siegel, R. (2016). Large contests. Econometrica, 84(2), 835-854.

[5] Olszewski, W., \& Siegel, R. (2020a). Performance-Maximizing Large Contests with Wojciech Olszewski. Theoretical Economics, 15(1), 57-88.

[6] Olszewski, W., \& Siegel, R. (2020b). Bid Caps in Large Contests. Games and Economic Behavior, forthcoming.

[7] Olszewski, W., \& Siegel, R. (2020c). Large Contests without Single Crossing. Economic Theory, forthcoming. 


\section{Appendix A}

Proof of Lemma 1. Starting from the initial condition $f_{T}(X)=X$, and working backward with (2) all the way to $f_{0}$, one can retrieve the whole sequence of functions $f$ for each of the eight possible ways to temporally allocate the four players. Finally, the equilibrium $X$ is the highest root in $[0,1]$ of $f_{0}(X)$. We derive in what follows $X^{*}$ for every temporal structure with four players, case by case.

If $n_{T}=4, f_{T-1}(X)=X(4 X-3)$. Then, $X^{*}(4)=3 / 4$.

If $n_{T}=3, f_{T-1}(X)=X(3 X-2)$, and hence $n_{T-1}=1, f_{T-2}(X)=$ $X^{2}(6 X-5)$. Then, $X^{*}(1,3)=5 / 6$.

If $n_{T}=2, f_{T-1}(X)=X(2 X-1)$, and

$\rightarrow n_{T-1}=2, f_{T-2}(X)=X\left(8 X^{2}-8 X+1\right)$. Then, $X^{*}(2,2)=$ $\sqrt{2} / 4+1 / 2$.

$\rightarrow n_{T-1}=1, f_{T-2}(X)=X^{2}(4 X-3)$, and hence $n_{T-2}=1$ and $f_{0}(X)=X^{2}\left(12 X^{2}-14 X+3\right)$. Then, $X^{*}(1,1,2)=\sqrt{13} / 12+7 / 12$.

If $n_{T}=1, f_{T-1}(X)=X^{2}$, and

$\rightarrow n_{T-1}=3, f_{T-2}(X)=X^{2}(6 X-5)$. Then, $X^{*}(3,1)=5 / 6$.

$\rightarrow n_{T-1}=2, f_{T-2}(X)=X^{2}(4 X-3)$, and $\rightarrow n_{T-2}=1, f_{T-3}(X)=X^{2}\left(12 X^{2}-14 X+3\right)$. Then, $X^{*}(1,2,1)=$ $\sqrt{13} / 12+7 / 12$.

$\rightarrow n_{T-1}=1, f_{T-2}(X)=X^{2}(2 X-1)$, and hence $n_{T-2}=2, f_{T-3}(X)=$ $X^{2}\left(12 X^{2}-14 X+3\right)$. Then, $X^{*}(2,1,1)=\sqrt{13} / 12+7 / 12$.

$\rightarrow n_{T-2}=1, f_{T-3}(X)=X^{2}\left(6 X^{2}-6 X+1\right)$, and hence $n_{T-3}=1$, $f_{T-4}(X)=X^{2}\left(24 X^{3}-36 X^{2}+14 X-1\right)$. Then, $X^{*}(1,1,1,1)=\sqrt{6} / 6+1 / 2$.

Proof of Lemma 2. In this Lemma we use formula (5) together with the functions $f$ and the equilibrium $X^{*}$ in Lemma 1 so as to characterized $W E$ for 
each of the 8 possible ways to temporally allocate the 4 players.

$$
\begin{aligned}
W E(4) & =4(1-X)^{2} X=\frac{3}{16}=0.1875 \\
W E(1,3) & =X(X-1)^{2}\left(36 X^{2}-24 X+7\right)=\frac{5}{18}=0.27778 \\
W E(1,1,2) & =X(X-1)^{2}\left(144 X^{4}-144 X^{3}+52 X^{2}-8 X+3\right)=\frac{163-5 * \sqrt{13}}{486}=0.29830 \\
W E(2,2) & =4 X(X-1)^{2}\left(8 X^{2}-4 X+1\right)=\frac{1}{4}=0.25 \\
W E(3,1) & =X\left(12 X^{2}+1\right)(X-1)^{2}=\frac{35}{162}=0.21605 \\
W E(2,1,1) & =X(X-1)^{2}\left(72 X^{4}-48 X^{3}+12 X^{2}+1\right)=\frac{193+7 * \sqrt{13}}{864}=0.25259 \\
W E(1,1,1,1) & =X(X-1)^{2}\left(576 X^{6}-864 X^{5}+456 X^{4}-96 X^{3}+12 X^{2}+1\right)=\frac{81+19 * \sqrt{6}}{432}=0.29523 \\
W E(1,2,1) & =X(X-1)^{2}\left(144 X^{4}-144 X^{3}+44 X^{2}+1\right)=\frac{65-\sqrt{13}}{216}=0.28423
\end{aligned}
$$

Proof of Lemma 3. In a fully simultaneous contest, from (7),

$$
\begin{aligned}
W E^{\infty}(\hat{n}) & =\lim _{\hat{n} \rightarrow \infty}\left[\hat{n}\left(\frac{1}{\hat{n}+1}\right)^{2}\right] \\
& =\lim _{\hat{n} \rightarrow \infty}\left[\frac{\hat{n}}{(\hat{n}+1)^{2}}\right] \\
& =0,
\end{aligned}
$$

which concludes the proof.

Proof of Lemma 4. In a fully sequential contest where, since all $n_{s}$ 's are equal to 1 , from (7) we obtain

$$
x_{i}(1,1, . ., 1,1) \approx \frac{1}{2^{t}}
$$


From (10) we find that,

$$
\begin{aligned}
W E^{\infty}(1, . ., 1) & =\lim _{\hat{n} \rightarrow \infty}\left[\left(\frac{1}{2}\right)^{2}+\left(\frac{1}{2^{2}}\right)^{2}+\left(\frac{1}{2^{3}}\right)^{2}+. .\right] \\
& =\sum_{k=1}^{\infty} \frac{1}{2^{2 k}} \\
& =\frac{1}{3},
\end{aligned}
$$

which concludes the proof.

Proof of Proposition 5. Notice from (8) that $W E^{\infty}\left(n_{1}, n_{2}, n_{3}, ..\right)$ decreases in $n_{1}$ for every $\left(n_{2}, n_{3}, ..\right)$. Hence, a global maximum must have $n_{1}=1$. Fixing $n_{1}=1, W E^{\infty}\left(1, n_{2}, n_{3}, ..\right)$ decreases in $n_{2}$ for every $\left(n_{3}, n_{4}, ..\right)$. Hence, a global maximum must have $n_{2}=1$. And so on. One can then conclude that a global maximum is reached by a fully sequential contest, where $W E^{\infty}(1,1, . ., 1)=1 / 3$. Additionally, as $W E^{\infty}\left(n_{1}, n_{2}, n_{3}, ..\right) \geq 0$ by definition, the global minimum is achieved by a fully simultaneous contest, where $W E^{\infty}(\hat{n})=0$ by Lemma 3 . Therefore, all the other temporal structures have $W E^{\infty} \in[0,1 / 3]$.

Proof of Proposition 6, Proposition 7, and Lemma 8. In the main body.

Proof of Lemma 9. In order to show that $W E^{\infty}(1, . ., 1)<W E^{\infty}(1, . ., 1,2)$, we first consider the two formulae for a fixed $n$ :

$$
\begin{aligned}
W E^{\infty}(1, . ., 1) & =\lim _{n \rightarrow \infty} \frac{\sum_{j=1}^{n} \frac{1}{2^{2 j}}}{\sum_{k=1}^{n} \frac{1}{2^{k}}} \\
W E^{\infty}(1, . ., 1,2) & =\lim _{n \rightarrow \infty} \frac{\sum_{j=1}^{n-2} \frac{1}{2^{2 j}}+2 * \frac{1}{2^{2(n-2)} * 3^{2}}}{\sum_{k=1}^{n-2} \frac{1}{2^{k}}+2 * \frac{1}{2^{n-2} * 3}}
\end{aligned}
$$

where we used (10). Note that in this last expression the term $2^{n-2} * 3$ comes from the first $n-2$ periods having a unique player, so that $1+n_{s}=2$, and the final two periods having 2 players, so that $1+n_{s}=3$.

Let $S_{k}(n)=\sum_{z=1}^{n} \frac{1}{2^{z}}$ and $S_{j}(n)=\sum_{z=1}^{n} \frac{1}{2^{2 z}}$. Then $W E^{\infty}(1, . ., 1)<$ $W E^{\infty}(1, . ., 1,2)$ can be restated as

$$
S_{j}(n)\left(S_{k}(n-2)+\frac{2}{3 \cdot 2^{n-2}}\right)<S_{k}(n)\left(S_{j}(n-2)+\frac{2}{\left(3 \cdot 2^{n-2}\right)^{2}}\right)
$$


which, after a few algebraic passages (see Appendix B.1), reduces to

$$
S_{k}(n-2) \cdot \frac{13}{2^{n}}+\frac{24}{2^{2 n}}<S_{j}(n-2) \cdot 3 .
$$

As we have $S_{k}(n-2)<S_{k}(\infty)=\frac{\frac{1}{2}}{1-\frac{1}{2}}=1$ and $S_{j}(n-2)>S_{j}(1)=\frac{1}{4}$ we see that as soon as $n \geq 5$, we obtain

$$
S_{k}(n-2) \cdot \frac{13}{2^{n}}+\frac{24}{2^{2 n}}<\frac{13}{2^{n}}+\frac{24}{2^{2 n}}<\frac{1}{4} \cdot 3<S_{j}(n-2) \cdot 3,
$$

and the extremes of (13) establish (12).

Proof of Lemma 10. In order to show that $W E^{\infty}(1, . ., 2)<W E^{\infty}(1, . ., 1,3)$, one could follow similar steps to the ones of the proof of Lemma 10 and show that

$$
\begin{aligned}
W E(1, . ., 1,2) & <W E(1, . ., 1,3) \\
& \Longleftrightarrow \frac{\sum_{j=1}^{\hat{n}-2} \frac{1}{2^{2 j}}+2 * \frac{1}{2^{2(\hat{n}-2)} * 3^{2}}}{\sum_{k=1}^{\hat{n}-2} \frac{1}{2^{k}}+2 * \frac{1}{2^{\hat{n}-2} * 3}}<\frac{\sum_{j=1}^{\hat{n}-3} \frac{1}{2^{2 j}}+3 * \frac{1}{2^{2(\hat{n}-3)} * 4^{2}}}{\sum_{k=1}^{\hat{n}-3} \frac{1}{2^{k}}+3 * \frac{1}{2^{\hat{n}-3} * 4}} \\
& \Longleftrightarrow \quad 2^{\hat{n}}\left(-2+2^{\hat{n}}\right)\left(-4+3 * 2^{\hat{n}}\right)\left(40-17 * 2^{\hat{n}+1}+4^{\hat{n}}\right)>0
\end{aligned}
$$

which is true for $\hat{n}>\frac{\log (17+\sqrt{249})}{\log (2)} \approx 5.03$, and hence in large contests.

Proof of Proposition 11. Throughout the proof, there are three algebraic passages which we store at the end of the proof; those to derive (14), (15) and (16).

Keeping the same number of total players $\hat{n}$, after a few algebraic passages (note for the referee: see below) we obtain that

$$
W E^{\hat{n}}(1, . ., 1, m)>W E^{\hat{n}}(1, . ., 1, m+1)
$$

is equivalent to (see Appendix B.2),

$S_{k}(\hat{n}-m-1) 2^{\hat{n}-m-1} \frac{5 m+3 m^{2}+m^{3}+4}{(m+1)(m+2)}+\frac{m+m^{2}+1}{m+2}>S_{j}(\hat{n}-m-1) 2^{2(\hat{n}-m)-1}$.

Consider now any $\hat{n} \geq 5$. Note that $W E^{\hat{n}}$ increases in $m$ for low $m$, as (14) 
fails if $m=1$, since we have

$$
\begin{aligned}
S_{k}(\hat{n}-2) 2^{\hat{n}-2} \frac{13}{6}+1 & <S_{k}(\infty) 2^{\hat{n}-2} \frac{13}{6}+1 \\
& =2^{\hat{n}-2} \frac{13}{6}+1 \\
& <\frac{1}{4} 2^{2 \hat{n}-3} \\
& =S_{j}(1) 2^{2 \hat{n}-3} \\
& <S_{j}(\hat{n}-2) 2^{2 \hat{n}-3}
\end{aligned}
$$

where the second inequality holds if $2^{\hat{n}}>\frac{26+2 \sqrt{241}}{3} \approx 19.02$, and hence it holds when $\hat{n} \geq 5$.

Furthermore, $W E^{\hat{n}}$ decreases in $m$ for high $m$, as (14) is satisfied if $m=\hat{n}-2$, since we have

$$
\begin{aligned}
& S_{k}(1) 2 \frac{5(\hat{n}-2)+3(\hat{n}-2)^{2}+(\hat{n}-2)^{3}+4}{(\hat{n}-1) \hat{n}}+\frac{(\hat{n}-2)+(\hat{n}-2)^{2}+1}{\hat{n}} \\
= & 2 \hat{n}-5+\frac{1}{\hat{n}-1}+\frac{5}{\hat{n}} \\
> & 2 \hat{n}-5 \\
\geq & 5 \\
> & S_{j}(1) 2^{3} .
\end{aligned}
$$

where the second inequality follows from $\hat{n} \geq 5$.

We now show that $W E^{\hat{n}}$ is single-peaked in $m$ by showing that if $W E^{\hat{n}}$ decreases in $m$ at some $m$, then it does so at $m+1$ as well. In other words, we want to show that if (14) holds at $m$, then it holds also at $m+1$. Algebraic passages (see Appendix B.3) show that (14) holds at $m+1$ if and only if

$S_{k}(\hat{n}-m-1) 2^{\hat{n}-m} \frac{14 m+6 m^{2}+m^{3}+13}{(m+2)(m+3)}+\frac{1}{2} \frac{\left(4 m+m^{2}+5\right)(m+1)}{(m+2)(m+3)}>S_{j}(\hat{n}-m-1) 2^{2(\hat{n}-m)-1}$

Notice that the right-hand side of (15) is identical to the right-hand side of (14). Next, we show that the left-hand side of (15) is strictly greater than the left-hand side of (14), thus proving the recursive claim.

We can rewrite the left-hand side of (15) as

$$
2\left(S_{k}(\hat{n}-m-1) 2^{\hat{n}-m-1} \frac{14 m+6 m^{2}+m^{3}+13}{(m+2)(m+3)}\right)+\frac{1}{2} \frac{\left(4 m+m^{2}+5\right)(m+1)}{(m+2)(m+3)}
$$


and, by $S_{k}(\hat{n}-m-1) 2^{\hat{n}-m-1}>S_{k}(\hat{n}-(\hat{n}-2)-1) 2^{\hat{n}-(\hat{n}-2)-1}=1$ which holds by $m<\hat{n}-2$, it is greater than

$$
S_{k}(\hat{n}-m-1) 2^{\hat{n}-m-1} \underbrace{\frac{14 m+6 m^{2}+m^{3}+13}{(m+2)(m+3)}}_{>\frac{5 m+3 m^{2}+m^{3}+4}{(m+1)(m+2)}}+\underbrace{\frac{14 m+6 m^{2}+m^{3}+13}{(m+2)(m+3)}+\frac{1}{2} \frac{\left(4 m+m^{2}+5\right)(m+1)}{(m+2)(m+3)}}_{>\frac{m+m^{2}+1}{m+2}},
$$

hence showing that the left-hand side of (15) is strictly greater than the lefthand side of (14), which concludes the recursive claim. Therefore, $W E^{\hat{n}}$ is single-peaked in $m$.

The optimal $m$ is found for the lowest $m$ such that (14) fails at $m$, but is satisfied at $m+1$. Ignoring integer constraints,

$$
\begin{aligned}
W E^{\hat{n}}(1, . ., 1, m) & =W E^{\hat{n}}(1, . ., 1, m+1) \\
& \Longleftrightarrow \frac{\sum_{j=1}^{n} \frac{1}{2^{2 j}}+\frac{m}{(m+1)^{2}} \frac{1}{2^{2(\hat{n}-m)}}}{\sum_{k=1}^{\hat{n}-m} \frac{1}{2^{k}}+\frac{m}{m+1} \frac{1}{2^{\hat{n}-m}}}=\frac{\sum_{j=1}^{\hat{n}-m-1} \frac{1}{2^{2 j}}+(m+1) * \frac{1}{2^{2(\hat{n}-m-1)} *(m+2)^{2}}}{\sum_{k=1}^{\hat{n}-m-1} \frac{1}{2^{k}}+(m+1) * \frac{1}{2^{\hat{n}-m-1} *(m+2)}}
\end{aligned}
$$

One can derive (see Appendix B.4) that the above-displayed equation is equivalent to

$$
\frac{2^{2 \hat{n}}(1+m)^{2}-2^{2 m}\left(1-m+m^{2}\right)}{3(1+m)\left(2^{\hat{n}}(1+m)-2^{m}\right)}=\frac{2^{2 \hat{n}}(2+m)^{2}-2^{2 m+2}\left(1+m+m^{2}\right)}{3(2+m)\left(2^{\hat{n}}(2+m)-2^{m+1}\right)} .
$$

Cross-multiplying and canceling out the terms which are identical on the right- and left-hand sides, we obtain the following polynomial degree 2 in $2^{\hat{n}-m}$ of the form $a * 2^{2(\hat{n}-m)}+b * 2^{\hat{n}-m}+c=0$, with

$$
\begin{aligned}
a & =(1+m)(2+m) \\
b & =-3\left(4+5 m+3 m^{2}+m^{3}\right) \\
c & =2\left(5+m-m^{2}\right) .
\end{aligned}
$$

One root requires $m>\hat{n}$, which is impossible, while the other root is admissible; ${ }^{12}$

$2^{\hat{n}-m}=\frac{3\left(4+5 m+3 m^{2}+m^{3}\right)+\sqrt{64+192 m+313 m^{2}+294 m^{3}+163 m^{4}+54 m^{5}+9 m^{6}}}{2(m+1)(m+2)}$

\footnotetext{
${ }^{12}$ The other root is like (17) except with "-" in front of the square root, which could be shown to imply $2^{\hat{n}-m} \in(0,1)$, and hence that $\hat{n}-m<0$; a contradiction.
} 
Let (17) define the solution $m(\hat{n})$. First note that, as $\hat{n} \rightarrow \infty$, also $m(\hat{n})$ must become unbounded, otherwise the left-hand side of (17) would become infinity, while its right-hand side would not. This rules out the optimality of finite $m$ 's in large $(1, . ., 1, m)$ contests. Hence, considering that $m(\hat{n}) \rightarrow \infty$ and canceling out the low powers in (17) we obtain

$$
\lim _{\hat{n} \rightarrow \infty} \frac{2^{\hat{n}-m(\hat{n})}}{3 m(\hat{n})}=1 .
$$

We finally show that (18) implies that $\lim _{\hat{n} \rightarrow \infty} \frac{m(\hat{n})}{\hat{n}}=1$. By construction, $\lim _{\hat{n} \rightarrow \infty} \frac{m(\hat{n})}{\hat{n}} \in[0,1]$. But also, as

$$
1=\lim _{\hat{n} \rightarrow \infty} \frac{2^{\hat{n}-m(\hat{n})}}{3 m(\hat{n})}=\lim _{\hat{n} \rightarrow \infty} \frac{2^{1-\frac{m(\hat{n})}{\hat{n}}}}{\frac{3 m(\hat{n})}{2^{\hat{n}}}},
$$

if we suppose by contradiction that $\lim _{\hat{n} \rightarrow \infty} \frac{m(\hat{n})}{\hat{n}} \in[0,1)$, then the above displayed equation implies

$$
\lim _{\hat{n} \rightarrow \infty} \frac{3 m(\hat{n})}{2^{\hat{n}}}>0 .
$$

But, since in the limit "exponentials are stronger than polynomials" and $m(\hat{n}) \leq$ $\hat{n}$, this is impossible. 


\section{Appendix B}

\section{Appendix B.1}

Algebraic passages from (11) to (12).

$$
\begin{aligned}
& S_{j}(n)\left(S_{k}(n-2)+\frac{2}{3 \cdot 2^{n-2}}\right)<S_{k}(n)\left(S_{j}(n-2)+\frac{2}{\left(3 \cdot 2^{n-2}\right)^{2}}\right) \\
& \left(S_{j}(n-2)+\left(\frac{1}{2^{2(n-1)}}+\frac{1}{2^{2 n}}\right)\right)\left(S_{k}(n-2)+\frac{2}{3 \cdot 2^{n-2}}\right) \\
& <\left(S_{k}(n-2)+\left(\frac{1}{2^{n-1}}+\frac{1}{2^{n}}\right)\right)\left(S_{j}(n-2)+\frac{2}{\left(3 \cdot 2^{n-2}\right)^{2}}\right) \\
& \left(S_{j}(n-2)+\frac{5}{2^{2 n}}\right)\left(S_{k}(n-2)+\frac{2}{3 \cdot 2^{n-2}}\right) \\
& <\left(S_{k}(n-2)+\frac{3}{2^{n}}\right)\left(S_{j}(n-2)+\frac{2}{\left(3 \cdot 2^{n-2}\right)^{2}}\right) \\
& S_{j}(n-2)\left(S_{k}(n-2)+\frac{2}{3 \cdot 2^{n-2}}\right)+\frac{5}{2^{2 n}}\left(S_{k}(n-2)+\frac{2}{3 \cdot 2^{n-2}}\right) \\
& <S_{k}(n-2)\left(S_{j}(n-2)+\frac{2}{\left(3 \cdot 2^{n-2}\right)^{2}}\right)+\frac{3}{2^{n}}\left(S_{j}(n-2)+\frac{2}{\left(3 \cdot 2^{n-2}\right)^{2}}\right) \\
& S_{j}(n-2) \frac{2}{3 \cdot 2^{n-2}}+\frac{5}{2^{2 n}} S_{k}(n-2)+\frac{5}{2^{2 n}} \frac{2}{3 \cdot 2^{n-2}} \\
& <S_{k}(n-2) \frac{2}{\left(3 \cdot 2^{n-2}\right)^{2}}+\frac{3}{2^{n}} S_{j}(n-2)+\frac{3}{2^{n}} \frac{2}{\left(3 \cdot 2^{n-2}\right)^{2}} \\
& \frac{S_{j}(n-2)}{3 \cdot 2^{n-3}}+\frac{5 S_{k}(n-2)}{2^{2 n}}+\frac{5}{3 \cdot 2^{3 n-3}} \\
& <\frac{S_{k}(n-2)}{3^{2} 2^{2 n-5}}+\frac{3 S_{j}(n-2)}{2^{n}}+\frac{1}{3 \cdot 2^{3 n-5}} \\
& \frac{S_{j}(n-2)}{3} 2^{2 n}+5 S_{k}(n-2) 2^{n-3}+\frac{5}{3} \\
& <\frac{S_{k}(n-2)}{3^{2}} 2^{n+2}+3 S_{j}(n-2) 2^{2 n-3}+\frac{4}{3} \\
& 3 S_{j}(n-2) 2^{2 n}+45 S_{k}(n-2) 2^{n-3}+3 \\
& <S_{k}(n-2) 2^{n+2}+27 S_{j}(n-2) 2^{2 n-3} \\
& S_{k}(n-2)\left(45 \cdot 2^{n-3}-2^{n+2}\right)+3<S_{j}(n-2)\left(27 \cdot 2^{2 n-3}-3 \cdot 2^{2 n}\right) \\
& S_{k}(n-2) 2^{n} \frac{13}{8}+3<S_{j}(n-2) 2^{2 n} \frac{3}{8} \\
& S_{k}(n-2) \cdot \frac{13}{2^{n}}+\frac{24}{2^{2 n}}<S_{j}(n-2) \cdot 3
\end{aligned}
$$




\section{Appendix B.2}

Algebraic passages to derive (14).

$$
\begin{aligned}
& W E^{\hat{n}}(1, . ., 1, m) \quad>\quad W E^{\hat{n}}(1, . ., 1, m+1) \\
& \Longleftrightarrow \frac{\sum_{j=1}^{\hat{n}-m} \frac{1}{2^{2 j}}+m * \frac{1}{2^{2(\hat{n}-m)} *(m+1)^{2}}}{\sum_{k=1}^{\hat{n}-m} \frac{1}{2^{k}}+m * \frac{1}{2^{\hat{n}-m} *(m+1)}}>\frac{\sum_{j=1}^{\hat{n}-m-1} \frac{1}{2^{2 j}}+(m+1) * \frac{1}{2^{2(\hat{n}-m-1)} *(m+2)^{2}}}{\sum_{k=1}^{\hat{n}-m-1} \frac{1}{2^{k}}+(m+1) * \frac{1}{2^{\hat{n}-m-1} *(m+2)}} \\
& \Longleftrightarrow \frac{\sum_{j=1}^{\hat{n}-m-1} \frac{1}{2^{2 j}}+\frac{1}{2^{2(\hat{n}-m)}} \frac{m^{2}+3 m+1}{(m+1)^{2}}}{\sum_{k=1}^{\hat{n}-m-1} \frac{1}{2^{k}}+\frac{1}{2^{\hat{n}-m}} \frac{2 m+1}{m+1}}>\frac{\sum_{j=1}^{\hat{n}-m-1} \frac{1}{2^{2 j}}+(m+1) * \frac{1}{2^{2(\hat{n}-m-1)} *(m+2)^{2}}}{\sum_{k=1}^{\hat{n}-m-1} \frac{1}{2^{k}}+(m+1) * \frac{1}{2^{\hat{n}-m-1} *(m+2)}} \\
& \Longleftrightarrow \frac{S_{j}(\hat{n}-m-1)+\frac{1}{2^{2(\hat{n}-m-1)}} \frac{m^{2}+3 m+1}{4(m+1)^{2}}}{S_{k}(\hat{n}-m-1)+\frac{1}{2^{\hat{n}-m-1}} \frac{2 m+1}{2(m+1)}}>\frac{S_{j}(\hat{n}-m-1)+\frac{1}{2^{2(\hat{n}-m-1)}} \frac{m+1}{(m+2)^{2}}}{S_{k}(\hat{n}-m-1)+\frac{1}{2^{\hat{n}-m-1}} \frac{m+1}{(m+2)}} \\
& \left(S_{j}(\hat{n}-m-1)+\frac{1}{2^{2(\hat{n}-m-1)}} \frac{m^{2}+3 m+1}{4(m+1)^{2}}\right)\left(S_{k}(\hat{n}-m-1)+\frac{1}{2^{\hat{n}-m-1}} \frac{m+1}{(m+2)}\right) \\
& >\left(S_{k}(\hat{n}-m-1)+\frac{1}{2^{\hat{n}-m-1}} \frac{2 m+1}{2(m+1)}\right)\left(S_{j}(\hat{n}-m-1)+\frac{1}{2^{2(\hat{n}-m-1)}} \frac{m+1}{(m+2)^{2}}\right) \\
& S_{j}(\hat{n}-m-1)\left(S_{k}(\hat{n}-m-1)+\frac{1}{2^{\hat{n}-m-1}} \frac{m+1}{(m+2)}\right) \\
& +\frac{1}{2^{2(\hat{n}-m-1)}} \frac{m^{2}+3 m+1}{4(m+1)^{2}}\left(S_{k}(\hat{n}-m-1)+\frac{1}{2^{\hat{n}-m-1}} \frac{m+1}{(m+2)}\right) \\
& >S_{k}(\hat{n}-m-1)\left(S_{j}(\hat{n}-m-1)+\frac{1}{2^{2(\hat{n}-m-1)}} \frac{m+1}{(m+2)^{2}}\right) \\
& +\frac{1}{2^{\hat{n}-m-1}} \frac{2 m+1}{2(m+1)}\left(S_{j}(\hat{n}-m-1)+\frac{1}{2^{2(\hat{n}-m-1)}} \frac{m+1}{(m+2)^{2}}\right)
\end{aligned}
$$




$$
\begin{aligned}
& S_{j}(\hat{n}-m-1) \frac{1}{2^{\hat{n}-m-1}} \frac{m+1}{(m+2)} \\
& +\frac{1}{2^{2(\hat{n}-m-1)}} \frac{m^{2}+3 m+1}{4(m+1)^{2}}\left(S_{k}(\hat{n}-m-1)+\frac{1}{2^{\hat{n}-m-1}} \frac{m+1}{(m+2)}\right) \\
& >S_{k}(\hat{n}-m-1) \frac{1}{2^{2(\hat{n}-m-1)}} \frac{m+1}{(m+2)^{2}} \\
& +\frac{1}{2^{\hat{n}-m-1}} \frac{2 m+1}{2(m+1)}\left(S_{j}(\hat{n}-m-1)+\frac{1}{2^{2(\hat{n}-m-1)}} \frac{m+1}{(m+2)^{2}}\right) \\
& S_{j}(\hat{n}-m-1) \frac{m+1}{m+2}+\left(S_{k}(\hat{n}-m-1) \frac{1}{2^{\hat{n}-m-1}} \frac{m^{2}+3 m+1}{4(m+1)^{2}}+\frac{1}{2^{\hat{n}-m-1}} \frac{m+1}{m+2} \frac{1}{2^{\hat{n}-m-1}} \frac{m^{2}+3 m+1}{4(m+1)^{2}}\right) \\
& >S_{k}(\hat{n}-m-1) \frac{1}{2^{\hat{n}-m-1}} \frac{m+1}{(m+2)^{2}}+\left(S_{j}(\hat{n}-m-1) \frac{2 m+1}{2(m+1)}+\frac{1}{2^{2(\hat{n}-m-1)}} \frac{m+1}{(m+2)^{2}} \frac{2 m+1}{2(m+1)}\right) \\
& S_{k}(\hat{n}-m-1) \frac{1}{2^{\hat{n}-m-1}}\left(\frac{m^{2}+3 m+1}{4(m+1)^{2}}-\frac{m+1}{(m+2)^{2}}\right) \\
& +\frac{1}{2^{2(\hat{n}-m-1)}} \frac{1}{m+2}\left(\frac{m^{2}+3 m+1}{4(m+1)}-\frac{2 m+1}{2(m+2)}\right) \\
& >S_{j}(\hat{n}-m-1)\left(\frac{2 m+1}{2(m+1)}-\frac{m+1}{m+2}\right) \\
& S_{k}(\hat{n}-m-1) \frac{1}{2^{\hat{n}-m-1}}\left(\frac{1}{4}(m+1)^{-2}(m+2)^{-2}\left(5 m+3 m^{2}+m^{3}+4\right) m\right) \\
& +\frac{1}{2^{2(\hat{n}-m-1)}} \frac{1}{m+2} \frac{1}{4}(m+1)^{-1}(m+2)^{-1}\left(m+m^{2}+1\right) m \\
& >S_{j}(\hat{n}-m-1) \frac{1}{2}(m+1)^{-1}(m+2)^{-1} m \\
& S_{k}(\hat{n}-m-1)\left(2^{\hat{n}-m-1}\right) \frac{1}{2} \frac{\left(5 m+3 m^{2}+m^{3}+4\right)}{(m+1)(m+2)}+\frac{m+m^{2}+1}{m+2} \frac{1}{2}>S_{j}(\hat{n}-m-1)\left(2^{\hat{n}-m-1}\right)^{2} \\
& S_{k}(\hat{n}-m-1)\left(2^{\hat{n}-m-1}\right) \frac{\left(5 m+3 m^{2}+m^{3}+4\right)}{(m+1)(m+2)}+\frac{m+m^{2}+1}{m+2}>S_{j}(\hat{n}-m-1) 2\left(2^{\hat{n}-m-1}\right)^{2} \\
& S_{k}(\hat{n}-m-1)\left(2^{\hat{n}-m-1}\right) \frac{\left(5 m+3 m^{2}+m^{3}+4\right)}{(m+1)(m+2)}+\frac{m+m^{2}+1}{m+2}>S_{j}(\hat{n}-m-1) 2^{2(\hat{n}-m)-1}
\end{aligned}
$$

which is (14) . 


\section{Appendix B.3}

Algebraic passages to derive (15).

Recall (14) :

$S_{k}(\hat{n}-m-1)\left(2^{\hat{n}-m-1}\right) \frac{\left(5 m+3 m^{2}+m^{3}+4\right)}{(m+1)(m+2)}+\frac{m+m^{2}+1}{m+2}>S_{j}(\hat{n}-m-1) 2^{2(\hat{n}-m)-1}$

Substituting $m+1$ we obtain

$$
\begin{aligned}
& S_{k}(\hat{n}-(m+1)-1) 2^{\hat{n}-(m+1)-1} \frac{\left(5(m+1)+3(m+1)^{2}+(m+1)^{3}+4\right)}{((m+1)+1)((m+1)+2)}+\frac{\left((m+1)+(m+1)^{2}+1\right)}{((m+1)+2)} \\
& >S_{j}(\hat{n}-(m+1)-1) 2^{2(\hat{n}-(m+1))-1} \\
& \left(S_{k}(\hat{n}-m-1)-\frac{1}{2^{\hat{n}-m-1}}\right) 2^{\hat{n}-m-2} \frac{\left(5(m+1)+3(m+1)^{2}+(m+1)^{3}+4\right)}{((m+1)+1)((m+1)+2)}+\frac{\left((m+1)+(m+1)^{2}+1\right)}{((m+1)+2)} \\
& >\left(S_{j}(\hat{n}-m-1)-\frac{1}{4^{\hat{n}-m-1}}\right) 2^{2(\hat{n}-m)-3} \\
& S_{k}(\hat{n}-m-1) 2^{\hat{n}-m-2} \frac{\left(5(m+1)+3(m+1)^{2}+(m+1)^{3}+4\right)}{((m+1)+1)((m+1)+2)} \\
& -\frac{1}{2} \frac{\left(5(m+1)+3(m+1)^{2}+(m+1)^{3}+4\right)}{((m+1)+1)((m+1)+2)}+\frac{\left((m+1)+(m+1)^{2}+1\right)}{((m+1)+2)} \\
& >\left(S_{j}(\hat{n}-m-1) 2^{2(\hat{n}-m)-3}-\frac{1}{2}\right)
\end{aligned}
$$

$$
\begin{gathered}
S_{k}(\hat{n}-m-1) 2^{\hat{n}-m-2} \frac{14 m+6 m^{2}+m^{3}+13}{(m+2)(m+3)} \\
\frac{1}{2} \frac{\left(4 m+m^{2}+5\right)(m+1)}{(m+2)(m+3)} \\
>S_{j}(\hat{n}-m-1) 2^{2(\hat{n}-m)-3}
\end{gathered}
$$

$S_{k}(\hat{n}-m-1) 2^{\hat{n}-m} \frac{14 m+6 m^{2}+m^{3}+13}{(m+2)(m+3)}+\frac{1}{2} \frac{\left(4 m+m^{2}+5\right)(m+1)}{(m+2)(m+3)}>S_{j}(\hat{n}-m-1) 2^{2(\hat{n}-m)-1}$

which is $(15)$. 


\section{Appendix B.4}

Algebraic passages to derive (16). The LHS of (16) is

$$
\begin{aligned}
\frac{\sum_{j=1}^{\hat{n}-m} \frac{1}{2^{2 j}}+\frac{m}{(m+1)^{2}} \frac{1}{2^{2(\hat{n}-m)}}}{\sum_{k=1}^{\hat{n}-m} \frac{1}{2^{k}}+\frac{m}{m+1} \frac{1}{2^{\hat{n}-m}}} & =\frac{\frac{1}{3}-\frac{1}{3} \frac{1}{2^{2(\hat{n}-m)}}+\frac{m}{(m+1)^{2}} \frac{1}{2^{2(\hat{n}-m)}}}{1-\frac{1}{2^{\hat{n}-m}}+\frac{m}{m+1} \frac{1}{2^{\hat{n}-m}}} \\
& =\frac{1}{3(m+1)} \frac{(m+1)^{2}+\left(3 m-(m+1)^{2}\right) \frac{1}{2^{2(\hat{n}-m)}}}{(m+1)-\frac{1}{2^{\hat{n}-m}}} \\
& =\frac{1}{3(m+1) 2^{\hat{n}}} \frac{2^{2 \hat{n}}(m+1)^{2}-2^{2 m}\left(1+m^{2}-m\right)}{2^{\hat{n}}(m+1)-2^{m}},
\end{aligned}
$$

while its RHS is (16)

$$
\begin{aligned}
\frac{\sum_{j=1}^{\hat{n}-m-1} \frac{1}{2^{2 j}}+(m+1) * \frac{1}{2^{2(\hat{n}-m-1)} *(m+2)^{2}}}{\sum_{k=1}^{\hat{n}-m-1} \frac{1}{2^{k}}+(m+1) * \frac{1}{2^{\hat{n}-m-1} *(m+2)}} & =\frac{\frac{1}{3}-\frac{1}{3} \frac{1}{2^{2(\hat{n}-m-1)}}+\frac{(m+1)}{(m+2)^{2}} \frac{1}{2^{2(\hat{n}-m-1)}}}{1-\frac{1}{2^{\hat{n}-m-1}}+\frac{m+1}{m+2} \frac{1}{2^{\hat{n}-m-1}}} \\
& =\frac{1}{3(m+2)} \frac{(m+2)^{2}+\left(3(m+1)-(m+2)^{2}\right) \frac{1}{2^{2(\hat{n}-m-1)}}}{(m+2)-\frac{1}{2^{\hat{n}-m-1}}} \\
& =\frac{1}{3(m+2) 2^{\hat{n}}} \frac{2^{2 \hat{n}}(m+2)^{2}-\left(1+m+m^{2}\right) 2^{2(m+1)}}{2^{\hat{n}}(m+2)-2^{m+1}}
\end{aligned}
$$

